\title{
Three Results for Trees, Using Mathematical Induction
}

\author{
W. A. Horn \\ Institute for Basic Standards, National Bureau of Standards, Washington, D.C. 20234
}

(December 12, 1971)

\begin{abstract}
The paper illustrates the use of mathematical induction to extend results which are true for a line segment to trees. Three separate theorems are stated and proved, each of which has some importance in its own right.
\end{abstract}

Key words: Helly's theorem; mathematical induction; minimum-length coverings; node-covering; trees.

\section{Introduction}

This paper presents three theorems each of which extends to trees a result that is known or obvious for a line segment. While the theorems are of interest in themselves, it is the purpose of the paper to use them as examples of how the inductive process may be effective in proving theorems for trees or for graphs with treelike structures. Other examples of the application of induction to trees include the proof that a tree with $n$ nodes has $n-1$ arcs (cf. [5], ${ }^{1}$ p. 35), or a paper such as [4].

The definition of a tree which will be used throughout is the following:

A tree is a connected graph containing no cycles.

\section{A Minimal Node-Cover Problem for Trees}

This section deals with the following problem: Given a (finite) tree $T$ and a collection $\left\{T_{i}\right\}_{1}{ }^{m}$ of subtrees, find a set of nodes $P$ in $T$ of least cardinality, such that

$$
P \cap T_{i} \neq \phi
$$

for all $i$. This problem has been considered for more complex graphs than trees (cf. [1], for example), but the following recursion algorithm, applicable to trees, is particularly simple to use.

Algorithm. Consider any "tip" node (node of order 1), $x \in T$. Let $A_{x}$ consist of $x$ and its associated arc.

1. If there exists some $T_{j}=\{x\}$, take $x$ to be in $P$ and derive the remaining members of $P$ as the set of nodes which solve the problem for the tree $T^{\prime}=T-A_{x}$, with the collection of subtrees

$$
\left\{T_{i}: i=1,2, \ldots, m, x \notin T_{i}\right\} .
$$

2. If no $T_{i}=\{x\}$, find $P$ as a solution to the problem on the tree $T^{\prime}$ with the collection of subtrees $\left\{T_{i}{ }^{\prime}\right\}_{1}{ }^{m}$, where

$$
T_{i}{ }^{\prime}=T_{i}-A_{x}
$$

for each $i$.

AMS Subject Classification: 0540.

${ }^{1}$ Figures in brackets indicate the literature references at the end of this paper. 
THEOREM 2.1. The above algorithm produces a minimal set $\mathrm{P}$.

Proof. Consider first case 1 , where some $T_{j}=\{x\}$. Let $P^{\prime}$ be some solution to the reduced problem stated in the algorithm. If $P$ is any solution to the original problem, then clearly $x \in P$, while $P-\{x\}$ meets at least every $T_{i}$ not containing $x$. Thus by the optimality of $P$ and $P^{\prime}, P-\{x\}$ and $P^{\prime}$ both have the same cardinality. Clearly $\{x\} \cup P^{\prime}$ is a feasible solution for the original problem.

In the second case, if $x \in T_{i}$ for some $i$, then each such $T_{i}$ must contain some element besides $x$. Since each such $T_{i}$ is connected, it must therefore contain $y$, the node at the other end of the arc containing $x$, since $y$ cuts $A_{x}$ from the rest of the tree $T$.

Now let $P^{\prime}$ be some optimal solution set of nodes for the reduced problem of case 2 . Since $T_{i}{ }^{\prime} \cap P^{\prime} \neq \phi$ for each $i, T_{i} \cap P^{\prime} \neq \phi$ also, so that $P^{\prime}$ is feasible for the original problem. Let $P$ be any optimal node set for the original problem. If $x \notin P$, then $P$ is a feasible solution for the reduced problem, so that $|P| \geqslant\left|P^{\prime}\right|$, proving the optimality of $P^{\prime}$. On the other hand, if $x \epsilon P$, consider the new node set

$$
P_{1}=P-\{x\} \cup\{y\}
$$

Since $P \cap T_{i} \neq \phi$ for each $i$, it follows that $P_{1} \cap T_{i} \neq \phi$ for each $i$, since if $x \in T_{i}$ then $y \epsilon T_{i}$ for any $i$. But again $P_{1}$ is a feasible solution for the reduced problem, so that

$$
|P|=\left|P_{1}\right| \geqslant\left|P^{\prime}\right|
$$

proving the optimality of $P^{\prime}$.

\section{Minimum-Length Coverings by Intersecting Subgraphs}

This section deals with an extension of a problem previously considered by the author [2].

Let $G$ be an undirected, connected graph whose edges have nonnegative lengths. If $A$ and $B$ are disjoint subgraphs of $G$, we shall use the notation $P(A, B)$ to refer to a (any) path in $G$ with one endpoint in $A$, the other in $B$, and no other point in $A \cup B$.

Let $\left\{S_{i}\right\}_{1}{ }^{n}$ be a collection of connected nonempty subgraphs of $G$ with the property that if $S_{i} \cap S_{i+1}=\phi$, for any $i$, then there exists a unique path $P\left(S_{i}, S_{i+1}\right)$. A collection $\left\{T_{i}\right\}_{1}{ }^{n}$ of connected subgraphs of $G$ will be called feasible, relative to $\left\{S_{i}\right\}_{1}{ }^{n}$, if

$$
\begin{array}{rlrl}
S_{i} \subset T_{i} & i=1,2, \ldots, n, \\
T_{i} \cap T_{i+1} \neq \phi^{\prime} & i=1,2, \ldots, n-1 .
\end{array}
$$

We treat the problem of choosing among feasible $\left\{T_{i}\right\}_{1}{ }^{n}$ so as to

$$
\operatorname{minimize} \sum_{i=1}^{n}\left|T_{i}\right|
$$

where $\left|T_{i}\right|$ is the sum of the lengths of all edges in $T_{i}$. A simple algorithm solves this problem. (The problem considered in [2] was the same as that treated here, except that compact intervals on the real line replaced the subgraphs $T_{i}$, above.)

Algorithm.

1. Let $T_{1}=S_{1}$.

2. Having determined $T_{k}$, find $T_{k+1}$ as follows.

(a) If $S_{k+1} \cap T_{k} \neq \phi$, set $T_{k+1}=S_{k+1}$.

(b) If $S_{k+1} \cap T_{k}=\phi$, let $P\left(S_{k+1}, T_{k}\right)$ be any path between $S_{k+1}$ and $T_{k}$. Set $T_{k+1}=S_{k+1} \cup$ $P\left(S_{k+1}, T_{k}\right)$.

THEOREM 3.1. The algorithm gives a minimal value for $\Sigma\left|T_{i}\right|$. 
Proof. The proof is by induction on the number $n$ of subgraphs $S_{i}$. Clearly the algorithm works for one subgraph. Assuming it works for $n-1$ subgraphs, we prove its validity for $n$ subgraphs.

First we show that there exists an optimal feasible solution $\left\{T_{i}\right\}_{1}{ }^{n}$, which minimizes $\Sigma\left|T_{i}\right|$, for which $T_{1}=S_{1}$. For if $\left\{T_{i}{ }^{\prime}\right\}_{1}{ }^{n}$ is optimal, but $T_{1}{ }^{\prime} \neq S_{1}$, let $P\left(S_{1}, T_{2}{ }^{\prime}\right)$ be any shortest path from $S_{1}$ to $T_{2}{ }^{\prime}$. Define a new collection of subgraphs

$$
\begin{aligned}
& T_{1}=S_{1}, \\
& T_{2}=T_{2}{ }^{\prime} \cup P\left(S_{1}, T_{2}{ }^{\prime}\right), \\
& T_{i}=T_{i}{ }^{\prime} \quad(3 \leqslant i \leqslant n) .
\end{aligned}
$$

Then it is clear that $S_{i} \subset T_{i}, 1 \leqslant i \leqslant n$, and that $T_{2}$ is connected, since $P\left(S_{1}, T_{2}{ }^{\prime}\right)$ is connected and intersects $T_{2}{ }^{\prime}$. Also, $T_{i} \cap T_{i+1} \neq \phi$, for all $i$, since $T_{1} \cap T_{2}$ contains the common point on $P\left(S_{1}, T_{2}{ }^{\prime}\right)$, and $T_{i} \cap T_{i+1} \subset T_{i}{ }^{\prime} \cap T^{\prime \prime}{ }_{i+1}$ for $2 \leqslant i \leqslant n$. Next we note that

$$
\left|P\left(S_{1}, T_{2}{ }^{\prime}\right)\right| \leqslant\left|T_{1}{ }^{\prime}-S_{1}\right|,
$$

for the connectivity of $T_{1}{ }^{\prime}$, together with the fact that $T_{1}{ }^{\prime} \cap T_{2}{ }^{\prime} \neq \phi$, imply that there exists a path from $S_{1}$ to $T_{2}{ }^{\prime}$ which is contained in $T_{1}{ }^{\prime}$ but does not contain any arcs of $S_{1}$. Thus we have from (3.4) and (3.5),

$$
\begin{aligned}
\left|T_{1}\right|+\left|T_{2}\right| & \leqslant\left|S_{1}\right|+\left|T_{2}{ }^{\prime}\right|+\left|P\left(S_{1}, T_{2}{ }^{\prime}\right)\right| \\
& \leqslant\left|S_{1}\right|+\left|T_{2}{ }^{\prime}\right|+\left|T_{1}{ }^{\prime}-S_{1}\right| \\
& =\left|T_{1}{ }^{\prime}\right|+\left|T_{2}{ }^{\prime}\right|,
\end{aligned}
$$

so that

$$
\sum_{i=1}^{n}\left|T_{i}\right| \leqslant \sum_{i=1}^{n}\left|T_{i}{ }^{\prime}\right|
$$

and the collection $\left\{T_{i}\right\}$ is optimal.

Now let $\left\{T_{i}\right\}_{1}{ }^{n}$ represent any optimal collection for which $T_{1}=S_{1}$, and let $\left\{T_{i}{ }^{*}\right\}_{1}{ }^{n}$ be a collection obtained from using the algorithm. We first note that $T_{2}{ }^{*} \subset T_{2}$. For if $S_{1} \cap S_{2} \neq \phi$, this is clear, since $T_{2}{ }^{*}=S_{2}$. If $S_{1} \cap S_{2}=\phi$, and $P\left(S_{1}, S_{2}\right)$ is the unique path from $S_{1}$ to $S_{2}$ in $G$, then $P\left(S_{1}, S_{2}\right) \subset T_{2}$, since if this were not the case then $T_{2}$ would be connected and contain $S_{2}$, while intersecting $\mathrm{S}_{1}$, so that there would exist another path in $T_{2}$ from $S_{2}$ to $S_{1}$, contradicting the uniqueness of $P\left(S_{1}, S_{2}\right)$. Since $T_{2}{ }^{*}=S_{2} \cup P\left(S_{1}, S_{2}\right)$, by definition, $T_{2}{ }^{*} \subset T_{2}$.

Thus the members of the collection $\left\{T_{i}\right\}_{2}{ }^{n}$ cover the respective members of the collection $\left\{T_{2}{ }^{*}, S_{3}, S_{4}, \ldots, S_{n}\right\}$ in $G$. If there exists a unique path in $G$ between $T_{2}{ }^{*}$ and $S_{3}$ (or if $T_{2}^{*} \cap S_{3} \neq \phi$ ), then the induction hypothesis applies to the set $\left\{T_{i}{ }^{*}\right\}_{2}{ }^{n}$, which was obtained by using the algorithm on $\left\{T_{2}^{*}, S_{3}, S_{4}, \ldots, S_{n}\right\}$, a set of $n-1$ elements, so that we have

$$
\sum_{i=2}^{n}\left|T_{1} *\right| \leqslant \sum_{i=2}^{n}\left|T_{i}\right|
$$

Since $\left|T_{1}\right|=\left|T_{1}{ }^{*}\right|$, the proof is complete once it is shown that any path from $T_{2}{ }^{*}$ to $S_{3}$ in $G$ is unique.

Assuming $T_{2}{ }^{*} \cap S_{3}=\phi$, let $P$ and $Q$ be two different paths from $T_{2}{ }^{*}$ to $S_{3}$ in $G$. Since $S_{2} \subset T_{2}{ }^{*}$, there exist paths $M$ and $N$ (possibly the same path) from $S_{2}$ to the points (possibly the same point) where $P$ and $Q$, respectively, meet $T_{2}{ }^{*}$. But then $M \cup P$ and $N \cup Q$ are different paths from $S_{2}$ to $S_{3}$, contrary to the initial assumption.

COROLlaRY 3.2. The algorithm is valid when $\mathrm{G}$ is a tree and the $\mathrm{S}_{i}$ are subtrees of $\mathrm{G}$.

Proof. We need only show that there exists a unique path between any two disjoint subtrees 
in a given tree. Suppose that there exist two distinct paths $P$ and $Q$ between subtrees $A$ and $B$, having respective endpoints $p_{A}$ and $q_{A}$ in $A$ and $p_{B}$ and $q_{B}$ in $B$. If $p_{A}=q_{A}$ and $p_{B}=q_{B}$, then $P=Q$, since there is a unique path between points in a tree. Therefore, assume $p_{A} \neq q_{A}$, and let $R_{A}$ be a path between $p_{A}$ and $q_{A}$ in $A$. If $P \cap Q \neq \phi$, then clearly $P \cup Q \cup R_{A}$ contains a cycle. If $P \cap Q=\phi$, let $R_{B}$ be a path between $p_{B}$ and $q_{B}$ in $B$. Then $P \cup Q \cup R_{A} \cup R_{B}$ is a cycle. Thus there exists a unique path between $A$ and $B$.

\section{Helly's Theorem for Trees}

Helly's theorem [2] states, in one dimension, that a collection of closed connected segments on a line $E^{1}$ has a nonempty intersection if and only if every pair of segments has a nonempty intersection. In this section we extend the result to trees.

THEOREM 4.1. A collection of subtrees of a tree has at least one common node if and only if every pair of subtrees has at least one common node.

Proof. The proof is by induction on the number of nodes. For a tree with 1 or 2 nodes the theorem is obvious.

Assume the theorem true for a tree with $n-1$ or fewer nodes and consider a tree $T$ with $n$ nodes. Let $S_{1}, S_{2}, \ldots, S_{m}$ be a collection of subtrees of $T$ such that

$$
S_{i} \cap S_{j} \neq \phi
$$

for every pair $(i, j)$.

Let $x$ be any node of $T$ which is also a cut point of $T$, and let $C_{1}, C_{2}, \ldots, C_{k}$ be the connected components of $T-\{x\}$. Let

$$
\begin{gathered}
A=\{x\} \cup C_{1}, \\
B=\{x\} \cup \bigcup_{i=2}^{k} C_{i} .
\end{gathered}
$$

Then $A$ and $B$ are subtrees of $T$. Furthermore, either

$$
S_{i} \cap A \neq \phi, \quad \text { all } i,
$$

or

$$
S_{i} \cap B \neq \phi, \quad \text { all } i .
$$

For if this were not the case, then we would have some $S_{i} \subset A-\{x\}$ and some $S_{j} \subset B-\{x\}$, so that $S_{i} \cap S_{j}=\phi$.

Suppose, without prejudice to the argument, that

$$
S_{i} \cap A \neq \phi, \quad \text { all } i,
$$

Let

$$
S_{i}{ }^{\prime}=S_{i} \cap A
$$

for each $i$. Then

$$
S_{i}{ }^{\prime} \cap S_{j}{ }^{\prime} \neq \phi,
$$

for every pair $(i, j)$. For if $S_{i}{ }^{\prime} \cap S_{j}{ }^{\prime}=\phi$ for some $(i, j)$, then since $S_{i} \cap S_{j} \neq \phi$ we must have a point $y \in B-\{x\}$ such that

$$
y \in S_{i} \cap S_{j}
$$

But since $x$ is a cut point and $S_{i} \cap A \neq \phi, S_{j} \cap A \neq \phi$, we must have $x \epsilon S_{i} \cap \dot{S}_{j}$, by the connectedness of $S_{i}$ and $S_{j}$. 
Thus $\left\{S_{i}{ }^{\prime}\right\}$ is a collection of pairwise intersecting subtrees of the finite tree $A$ of order less than $n$. By the induction hypothesis, there exists a point

$$
z \epsilon \bigcap_{i=1}^{m} S_{i}{ }^{\prime} \subset \bigcap_{i=1}^{m} S_{i}
$$

proving the theorem.

\section{References}

[1] Edmonds, J., Covers and packings in a family of sets, AMS Bull. 68, 494-499 (1962).

[2] Helly, E., Uber Mengen konvexer Korper mit gemeinschaftlichen Punkten, Jber. Deut. Math. Verein. 32, 175-176 (1923).

[3] Horn, W. A., Minimum-length covering by intersecting intervals, J. Res. Nat. Bur. Stand. (U.S.), 73B, No. 1, 49-51 (1969).
[4] Horn, W. A., Optimal design of sorting networks, submitted for publication.

[5] Ore, Oystein, Graphs and Their Uses, (Random House, 1963) (paperback).

(Paper 76B1\&2-359) 